\title{
Interactive on-line perception of line patterns
}

\author{
EDWARD L. MOROFSKY \\ Biomedical Computer Laboratory \\ Washington University School of Medicine \\ St. Louis, Missouri 63110
}

and

\author{
ANDREW K. C. WONG \\ Biotechnology Program, Carnegie-Mellon University \\ Pittsburgh, Pennsylvania 15213
}

A pattern perception system, PPS, has been implemented on the $360 / 67$ time-sharing system with the capability of analyzing, representing, comparing, and classifying complex line patterns. Patterns are input to the system as digitized arrays and are subject to tracing by several heuristic methods based on junction characteristics. The trace yields a set of component lines. The representation is a structured pattern description in terms of these components and distinguishes the topological structure of the pattern as a whole from the primitive features (straight segments, curves, angles) that constitute each line component of the pattern. The choice of a reference component about which to cluster the description is the determining factor in the structure obtained, and a simplicity criterion is advanced to identify a plausible first choice for this role. The topological structural description acts as the initial discriminator in the comparison or matching of patterns. Since the topological description is orientation, size, and feature invariant, such information need not be retrieved from the primitive feature portion of the description unless the initial comparison is positive. This form of representation facilitates the identification of transformationaily related patterns and eliminates the need for normalization of patterns. The comparison of topological structures begins at the reference component (Level 1) and proceeds from level to level (component levels are determined by their relationship to the reference). The level-by-level comparison is used to extract embedded subpatterns and serves as the basis for scene analysis in PPS. This organized structural representation is referred to as the perceived pattern and is the product of the constructive aspect of perception. All further processing achieved by PPS is accomplished through the use of this representation. Resulting descriptions can be reduced by compressing multiple examples of component interrelationships to their general recursive rule of formation. Such a reduction of recursive patterns to their common generative form is a prerequisite for efficient classification, i.e., patterns formed by or containing a random number of applications of some simple interrelationship are more easily recognized as similar when the common generation rules have been isolated. This process results in two different levels of pattern description-the unreduced specific description with all of its peculiarities and the reduced description of the general class of which it is a member. It is the general descriptions that are stored in a pattern universe as a memory of known patterns. Unknown input patterns are compared parallelly with those contained in the pattern universe and are either classified as members of a pattern universe class or used as the basis for the formation of a new class. Many of the methods used in PPS were based on findings in perceptual psychology, e.g., hierarchical representation and sequential comparison. The manner in which such concepts were incorporated into PPS and their further influences on the functioning of the system will be discussed.

A pattern perception system, PPS, has been implemented in the APL computer language (Falkoff \& Iverson, 1969) on the 360/67 time-sharing system with the capability of analyzing, representing, comparing, and classifying complex line patterns. The representational structures and processing methodologies of PPS were designed with the explicit objective of incorporating some known and hypothesized characteristics of human perception. This paper contains an elementary description of the principal system components along with the specific psychological evidence which served as the motivating influence on the design. The implications of these decisions in terms of system efficiency and performance are discussed. Complete technical system description can be found in Morofsky and Wong (1971), Morofsky (1972), Wong and Morofsky, ${ }^{1}$ Morofsky and Wong, ${ }^{2}$ and Wong and Morofsky. ${ }^{3}$

The initial goal of PPS is the analysis of the input pattern into its constituent line components and the generation of a structured description of the relationships among these components. The description is hierarchical with relationships defined between components of adjacent levels as well as the same level. The first level component serves as the reference about which the description is clustered. The resulting description is embodied in a flexible representational structure designed to facilitate the processing involved in the comparison and identification of line patterns and in the analysis of scenes. The design criteria of PPS result in a decreased efficiency in routine classification tasks, but allows a flexibility of representation and processing that more nearly parallel human performance in complex perceptual tasks.

Human pattern perception involves an interaction between the encoded representation of the visual scene and an internal representation of known pattern classes and concepts stored in memory. These representations are in a continuous state of refinement and decay as finer distinctions are drawn or as old concepts are no longer useful. Therefore, the visual input is used to update and expand continually the memory storage of known patterns, and these pattern classes are, in turn, used to analyze and classify the visual scene. An essential prerequisite of such interaction is an efficiently organized memory storage. It must facilitate retrieval of specific classes and accommodate the modification, addition, and deletion of classes. The memory storage of patterns must also be responsive to changing representations or refinements of a class over time.

The possible interactions between an unknown input pattern description and the system memory of known 


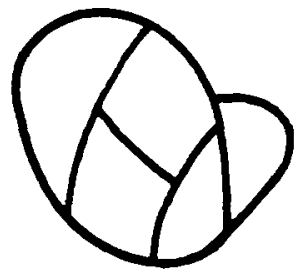

(a)

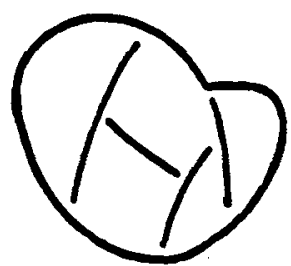

(c)

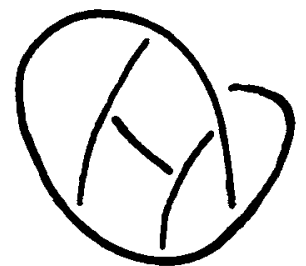

(i)

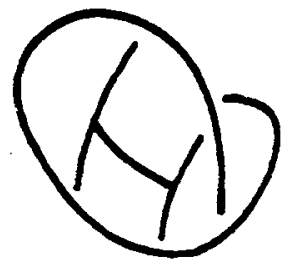

(d)
Fig. 1. Pattern of (a) with components obtained by a continuity trace (b), by an outer boundary trace (c), and in (d) by isolating a familiar subpattern "H." Representation of (d) is held to be a bater product of (b) or (c).

pattern classes, or $\mathrm{PU}$, define the basic implemented capabilities of PPS. Reduction of the search space of possible pattern matches, PU, to a subset containing only those classes that are potential matches is the first step. This reduction is based on partial cues, another human characteristic. Instead of matching the unknown with each potential pattern class in turn, PPS attempts to synthesize or replicate, in paralle!. each potential pattern class. The number of replications is continually decreased as the necessary essential components fail to be located in the input. This process eliminates the need to segment a complex scene before classifying the individual subpatterns and reduces scene analysis to a serial application of the simple pattern identification routine.

\section{DESCRIBING PATTERNS IN PPS}

The first choice to be made in the analysis of a line drawing is the method of decomposing the pattern into components. The method chosen will affect the types of structures obtained and the relations involved. A study by the psychologist Djang (1937) served as the starting point for component definition in PPS. The Ss in this study were asked to reconstruct meaningless patterns that had previously been presented to them and to indicate the perceptual units involved. The results seemed to indicate three main types of processing as shown in Fig. 1. The pattern is built up from line segments that close or terminate at junctions with other components (Fig. lb), much like one might draw the pattern without breaking contact between pencil and paper for each component, and no backtracking or retracing is allowed. The second type is exactly the same, except that an enclosing boundary. if one exists. is chosen as a component ( $\mathrm{Fig}$. $\mathrm{lc}$ ) and all inner lines are then completed. In the third type of reconstruction (Fig. 1d), the $S$ builds a structure around a subpattern which resembles a meaningful pattern. It was then necessary to ask whether memory does in fact influence the decomposition of an unknown pattern into a meaningful subpattern or if a representation in terms of a meaningful subpattern is the later product of higher-level processes on an ordinary decomposition of Type B or C. We decided on the latter interpretation. reasoning that a pattern or subpattern cannot in fact be recognized before it is represented in some encoded description. In the words of Merleau-Ponty (1962), "Before any contribution by memory, what is seen must at the present moment so organize itself as to present a picture to me in which I can recognize my former experiences." This view is in contradiction to the commonly held opinion that familiar patterns are easier to see, which gains support from the word-frequency effect or the inverse relation between the frequency of occurrence of a word in print and the viewing time required for its identification. However, the apparent rapidity with which familiar patterns can be identified may well be due to a partial match in which the missing portions of the percept or representation are filled in by the observer (Robinson, 1969). This led us to the establishment of two initial trace strategies in PPS-the continuity trace (Fig. 1b) and the outer boundary trace (Fig. 1c).

Once components have been obtained, one must decide upon the relational structure and the relations to he used. A representation will be the most important result of a pattern analysis and must contain, in explicit form, defining relational invariants of a pattern class and be of such a form that identifiable subpatterns can be extracted or added with minimal disruptions of the original description. Since various alternative descriptions are possible, the goal of the specific description is to organize the pattern components into a structure which contains their meaningful interrelationships. The core of the specific description is a hierarchical description suggested by the demands of efficient processing and the results of retinal stabilization studies (Evans, 1968; Heckenmueller. 1969: Pritchard, Heron, \& Hebb, 1960), in which the image of a visual target is stabilized on the retina. These studies show that fragmentation of patterns occurs with retinal stabilization. Perceived patterns break up into parts. some or all of which may disappear. Complex patterns seem to remain visible for a longer time. The lines comprising a complex figure behave independently of each other, and each separate element of the figure disappears and regenerates as a unit. The nature of the response to the physiological strain imposed by retinal stabilization provides insight into the manner in which patterns are analyzed and processed. It has been observed (Mackay, 1970) that the effects of the stabilized retinal image are transferred between eyes. indicating the presence of central as well as peripheral 

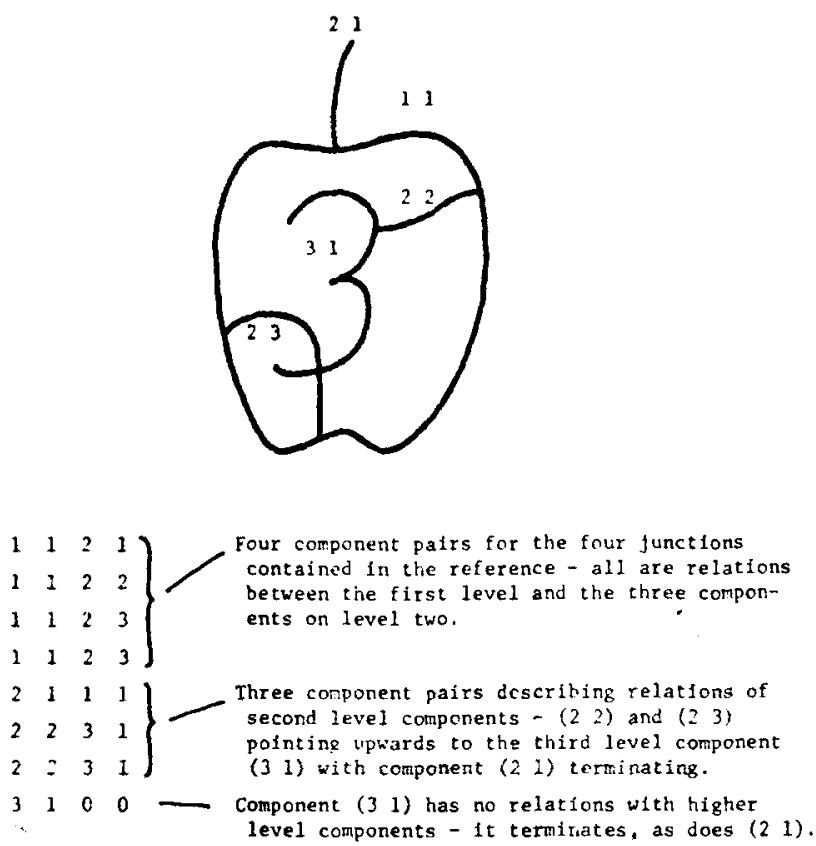

With component (1 1 ), the apple outline, acting as reference the average hierarchical level is 2, while with the " 3 " as reference the sverage hierarchical level would be 2.4 . The simplicity criterion '

Fig. 2. The pattern "apple-3" is shown with labeled components and the resulting linkage field of component pairs which is the basis of the pattern description in PPS.

factors. Thus, neural adaptation, as well as photochemical adaptation, is involved. The fragmentation is not haphazard, but occurs in such a way as to retain a meaningful pattern. In the words of one of the investigators (Evans, 1968), "... we have demonstrated that fragmentation phenomena are not solely a function of retinal stabilization and the hypothesis that the partial perception of patterns reflects the partial and variable activation of a perceptual hierarchy seems to be supported." The hierarchical description employed by PPS begins at the component or components on Level 1, with all other components assigned levels determined by their relationship to this first level. When the first level is occupied by one component, it is referred to as the reference component. All components sharing a junction with the reference are assigned hierarchical Level 2, and, in general, components are assigned a level of one more than the lowest level component with which they share a common junction. This process is indicated in Fig. 2, where the components have been labeled in this manner. The choice of a reference component naturally is the prime determinant of the structured description after the decomposition has been performed. A simplicity criterion, which selects the component that, when acting as reference, results in a hierarchical structure having the lowest average hierarchical level, is proposed to identify a plausible first choice for this role. The susceptibility to variation in the structured description (due to indecision as to the choice of reference) may be viewed as an indication of the instability of the organization of the perceived pattern. A pattern that can be represented by hierarchical structures of equal stability or simplicity is considered to be an ambiguous pattern. The perceived pattern may then shift from one representation to another. While the above scheme offers a method of measuring the relative complexities among various representations of the same pattern, the problem of ranking different patterns according to their complexity in agreement with human judgments of difficulty of reproduction remains unsolved. It requires a weighting of the feature primitives on each level in terms of the number of levels used and the types of relations among levels as a contribution to the total complexity of the figure.

Each component is individually analyzed into primitive features, namely, curved segments of both positive and negative curvature, straight segments, and angles. This is accomplished on the bearing string representation introduced by Freeman (1961), which approximates a component by a series of directed segments having one of the eight possible bearings shown in Fig. 3. Each component is described as a sequence of primitive features, i.e., straight and curved segments of various lengths, curvatures, and bearings. The main obstacle in analyzing components having multiple features is in deciding where the segmentation points between features occur and ensuring that the resulting features have some resemblance to those which human observers see. The segmentation points are determined in sequence as the features are synthesized. Each component of the hierarchy is tagged with a double level of description. The first simply indicates whether the component is closed and which primitive components it contains. A hash-coded representation in terms of the type, sequence, and dimensions of the features is also given. A hash table index is calculated, and the data can be retrieved from the hash table by the use of the index. Numerical vectors, matrices, and characters may all be hashed in this manner. It is the hash-code index which is

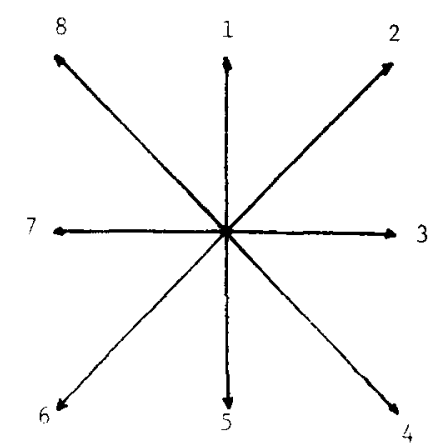

Fig. 3. The eight possible bearings of the directed line segments that are used to approximate the line components in PPS. 


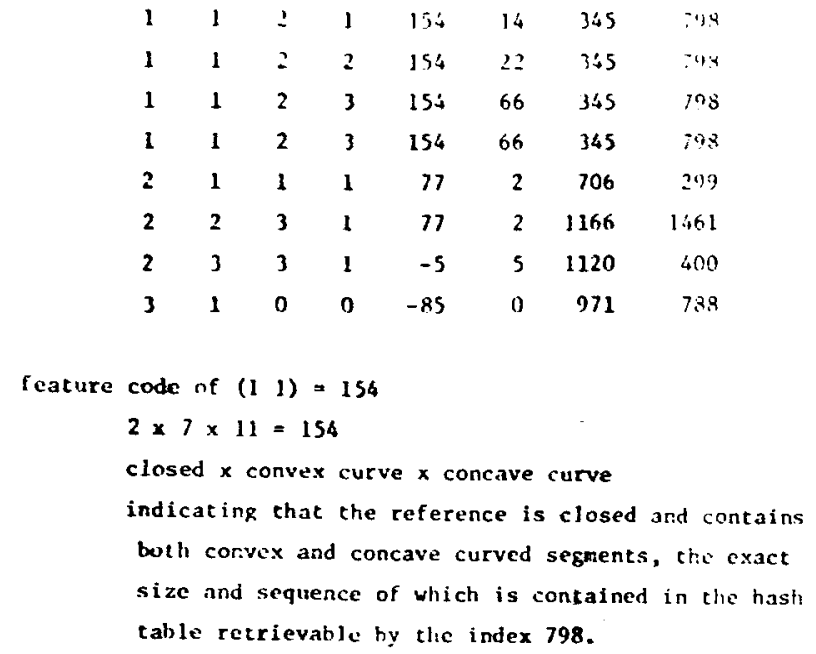

junction code describing the relation of components $(11)$ and (2 1) is equal to 14.

$2 \times 7=14$, which indicates that the apple stem (2 1) has a $T$-juncticn with the apple out 1 ine (1 1) and lies outside of (1 1 ).

Fig. 4. The description, D, of the "apple-3" drawing. The four-column linkage feld of Fig. 2 has been augmented by a feature code, junction code, and two hash codes in Columns 5 , 6,7 , and 8 , respectively.

stored in the description. A hash code is preferable to a list label in that a unique code can always be obtained from identical data without matching the entry data with all data previously stored in search of a common label. Thus, if one wishes to collect all known patterns having a closed reference component, it is not necessary to search the detailed description of each reference, but a simple examination of the partial features code is sufficient.

Each row of the specific description, D, describes a component and its relation to another component with which it shares a common junction. This relation is contained in a junction code and describes the type of junction and the relative position between the two components, which are referred to as a component pair. A complete description of the pattern of Fig. 2 is shown in Fig. 4.

\section{OPERATIONS ON REPRESENTATIONS}

The representation of the perceived pattern serves not only to structure the visual field in a meaningful way, but also, by its very construction, to lend itself to more efficient processing. The data structure of PPS can be extended or compressed by the insertion or deletion of component pairs as long as the resulting structure satisfies the requirements of a description (Wong \& Morofsky ${ }^{1}$ ). The first operation to be considered is the comparison of two descriptions with the objective of specifying the degree of their resemblance, if any.

The structure of the descriptive representations will obviously affect the type of matching algorithms that can be considered. This raises the familiar issue of whether visual recognition is a parallel, one-step process or a serial one. Experimental studies, in which the time taken by a $S$ to recognize different patterns is measured, have tended to support the serial recognition viewpoint. The $S$ is asked to scan a group of patterns, searching for a previously encountered pattern. It was found (Norton \& Stark, 1971) that the $S$ takes longer to recognize the previously encountered pattern than to reject an unfamiliar one. It was also found that the $S$ takes longer to recognize complex patterns than to recognize simple ones. Both results are to be expected if patterns are matched serially.

Pattern matching in PPS begins at the reference component and proceeds to greater levels of the hierarchy as long as a match is maintained. Various degrees of correspondence are available ranging from topological equivalency to identity. This simple matching procedure is utilized in classifying an unknown input pattern as a member of a known class in the memory or pattern universe, PU. A sample PU containing five pattern classes is shown in Fig. 5. The PU contains the defining representations of known pattern classes in an extendable array of 10 columns. The rows of the PU are grouped into cells, each of which represents a pattern class. A pattern class address vector, PCA, gives the row indices of the PU at which each pattern class cell begins. A pattern class name vector, $\mathrm{PCN}$, is used in conjunction with PCA and contains the hash-coded class name corresponding to each class address of PCA. The PCA and PCN for the sample PU are also given in Fig. 5. The first six columns of each cell are equivalent to the corresponding columns of the description $D$. The seventh column contains the recursive label, which is used in representing patterns containing recursive relations and will be discussed later. Column 9 indicates whether or not the component is essential to the class definition, while Column 8 registers the frequency of component presence during learning, thus providing a means of changing the status of the component as a result of experience (Wong \& Morofsky ${ }^{3}$ ).

If an element of Column 9 of the PU is 1 , then the corresponding component of Columns 1 and 2, say (i j), is essential and must be present in an unknown $D$ if the unknown pattern is to be classified as a member of the pattern class. If Column 9 is 0 , the component ( $\mathrm{ij}$ ) is nonessential and may be present in the $D$, but its presence is not necessary. It can be seen from Fig. 5 that in the pattern apple. the apple outline is essential to the class and the stem is not. Assignment of the stem as nonessential means that it will be seen as part of the apple when present rather than as a component external to the apple. This is an important concept for discerning objects embedded in a scene. An additional component, attached to an identified object and not corresponding to a nonessential component at its connection level, is considered to be external to the pattern.

A more complicated case of pattern classification 
Fig. 5. Sample PU representing the pattern classes, apple, “3," tree, house, and ship.

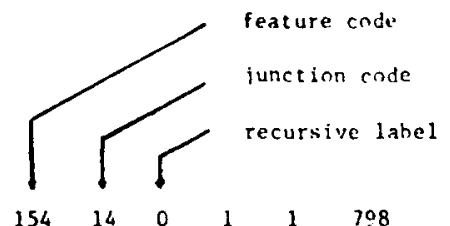

$\begin{array}{llll}1 & 1 & 2 & 1 \\ 2 & 1 & 1 & 1 \\ 1 & 1 & 0 & 0 \\ 1 & 1 & 2 & 1 \\ 1 & 1 & 2 & 2 \\ 2 & 2 & 1 & 1 \\ 1 & 1 & 2 & 1 \\ 1 & 1 & 2 & 2 \\ 1 & 1 & 2 & 2 \\ 1 & 1 & 2 & 1 \\ 2 & 1 & 1 & 1 \\ 2 & 2 & 1 & 1 \\ 1 & 1 & 2 & 1 \\ 1 & 1 & 2 & 1 \\ 2 & 1 & 3 & 1 \\ 2 & 1 & 3 & 1 \\ 3 & 1 & 2 & 1\end{array}$

occurs when more than one known pattern occurs in a description of an input pattern. A description containing multiple patterns is referred to as a scene. The classification of the constituent patterns of a scene is naturally a more difficult problem, since it involves both the segmentation of the scene into separate patterns and their assignment to pattern classes. Normally, this segmentation must be accomplished before classification can begin. However, PPS performs both the separation and classification simultaneously by using the analysis-by-synthesis approach. That is, an identifiable pattern is synthesized or constructed from components contained in D until a definite classification is made-this being the first step in the analysis of $D$ into its constituent patterns. Beginning with the reference component of $\mathrm{D}$. all possible matching pattern classes of the PU are synthesized. level by level, using the available components of $\mathrm{D}$ (initially only essential components). Pattern classes of the PU are eliminated when one of their essential components fails to find a match in D. All surviving pattern classes have a match in $\mathrm{D}$ according to the degree of correspondence specified. After a pattern class has been found to have its essential components matched in $\mathrm{D}$, the nonessential components of the class are matched with the remaining components of $\mathrm{D}$. All previously unmatched components of D that correspond

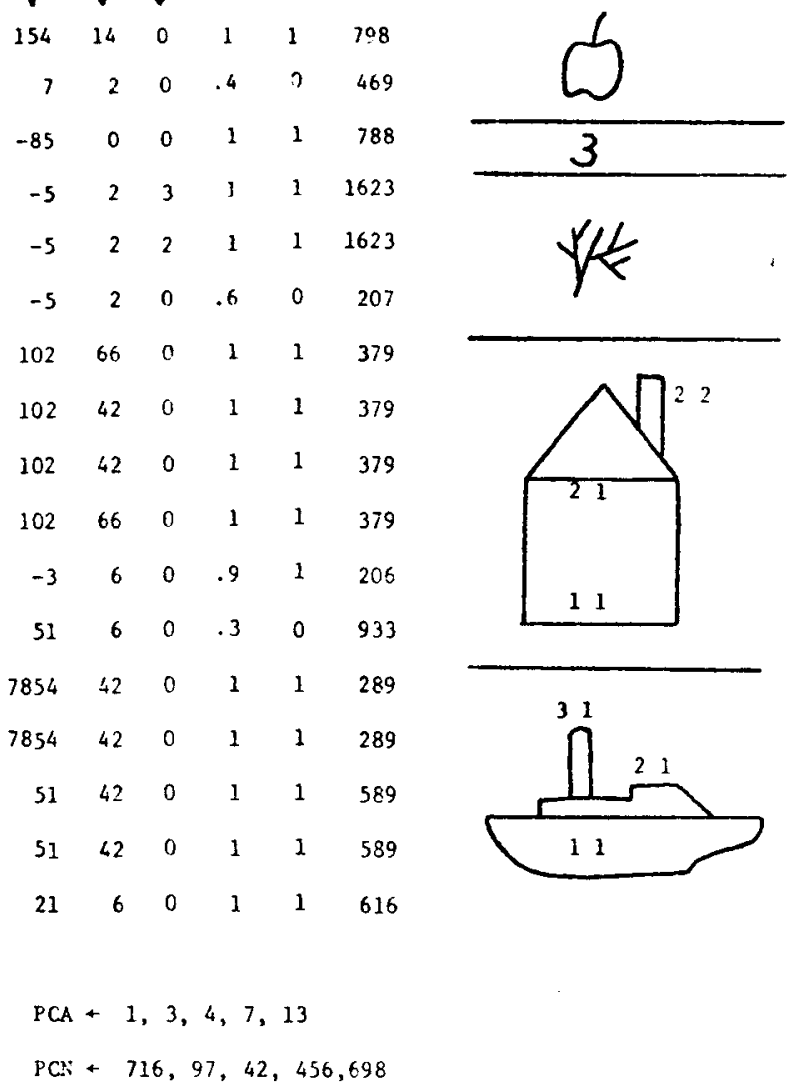

to nonessential components are added to the previously identified subpattern. If all components of the description are not contained in the first synthesis, the remaining components are regrouped into a new description and a second synthesis is begun. This process is continued until all components have been identified or found not to match any of the pattern classes in the PU. Thus, descriptions containing one pattern and those containing multiple patterns are treated in the same manner, and no special preprocessing is required.

The suggestion (Mackay, 1970) that analysis of complex scenes might well be advantageously accomplished by a process of internal replication-with the internal specification serving as a compact description of the pattern-has been utilized for scene analysis in PPS. The search space of possible pattern matches. PU, is reduced to a subset containing only those pattern classes which might possibly be synthesized from the reference of $D$. This reduction is performed according to the feature codes of the reference components and the levels of essential components. This replicating procedure is particularly useful. since it is unaffected by transformations that leave the pattern invariant and requires no modification when going from single pattern to complex scene. Such a "matching response" is much more general than that of 


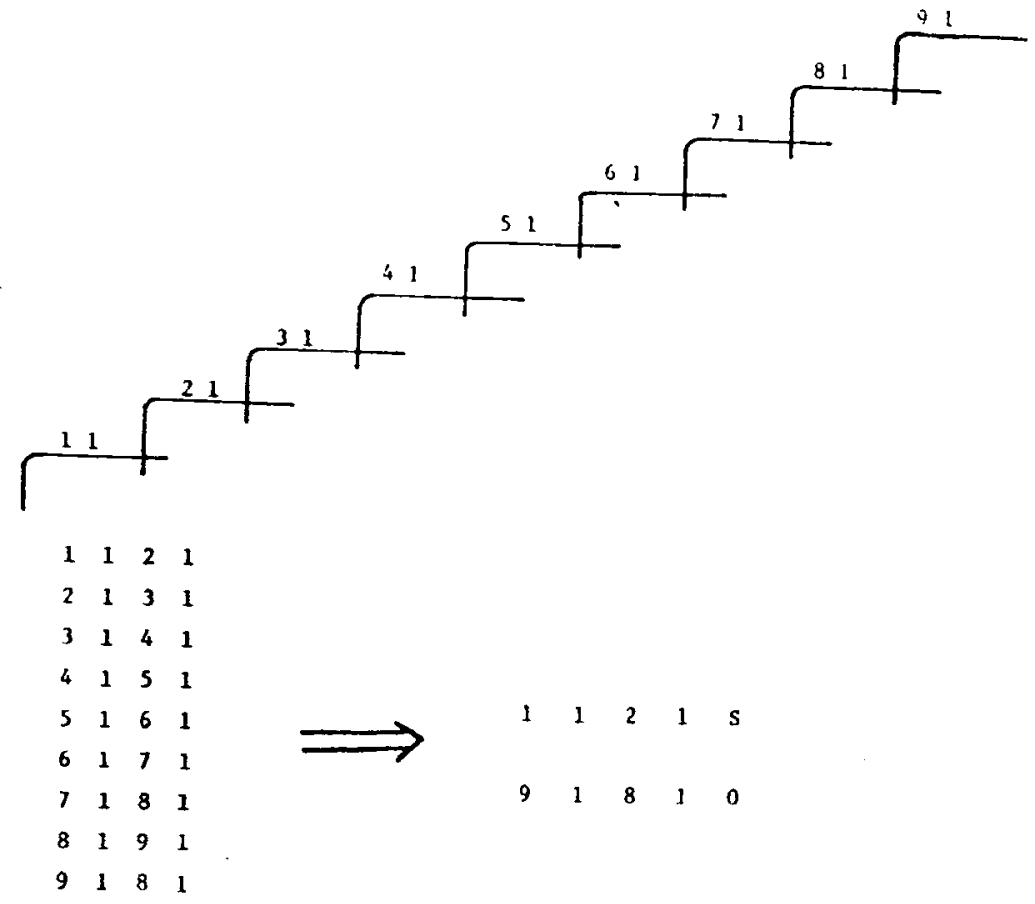

Fig. 6. A simple vertically recursive pattern with labeled components is shown along with the linkage field before and after compression to the recursive form. The $S$ in Column 5 of the compressed linkage field indicates simple vertical recursion.

simple imitation and makes particularly helpful the use of essential and nonessential pattern components. Such essential and nonessential components or relations give one the ability to differentiate the defining components and relations of a given class from those that may occur but whose presence is not necessary. For example. a tree must have branches but may or may not have leaves. Both are instances of the class tree

\section{MEMORY AND THE GENERAL CLASS}

The specific description, $D$, of an input pattern contains all of the information needed to specify uniquely the given pattern. Such a description can be used to reconstruct the pattern, to answer inquiries about its structure, or to compare different patterns. The pattern classes stored in the PU are general descriptions of groups of patterns sharing some common characteristics. The reduction of specific descriptions is accomplished by compressing multiple examples of component interrelationships to their general recursive rule of formation. Recursive patterns are composed, wholly or in part, of components exhibiting a distinctive series of repeating structural relations. Their representations present unique opportunities for a more compressed description and generality of pattern class representation. The binary tree structure, for example, is distinguished by the branching relation, which can be considered a simple vertical recursion, i.e., similar branching recurs indefinitely from one level to the next. Compound recursion in depth refers to a recurring sequence containing more than one relation. A vertically recursive pattern is identified, from an examination of its description $D$, by isolating an interconnected series of component pairs pointing from lesser to greater hierarchical levels and having a repetitive series of structural connections. The basic set of components whose structural relations are repeated is called a subuniverse, $\mathrm{SU}$, and its identification is the first step in recognizing a recursive pattern. Obviously, the original description must be structured in such a manner that the recursive relations are present. This requirement is only fulfilled by a proper component set and choice of reference.

The objective of efficient recursive pattern description is to compress the recursive portions of $D$ into a single relation (in simple vertical recursion) or a reduced set of relations (in compound vertical recursion). Compressed representations of recursive patterns save considerable space and achieve generality of pattern class description. Two basic types of recursion are treated by PPS, vertical
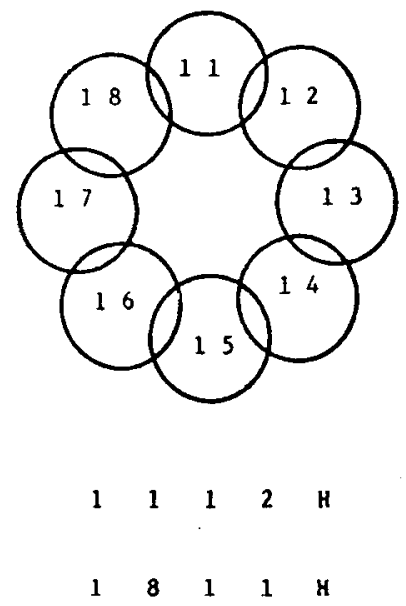

Fig. 7. A horizontally recursive pattern with eight first-level components. The compressed linkage field is given with the $\mathbf{H}$ representing horizontal recursion. 
and horizontal. Vertical recursion involves components of differing hierarchical levels which can be telescoped into a single set of defining relations. Horizontal recursion involves components of equal hierarchical level which are all similarly related, one to the other. An additional column is inserted into the description of compressed recursive patterns to indicate which component pairs exhibit recursive connections and what specific type of recursion is involved. These labels are indicated in Figs. 6 and 7. A detailed system for the realization of recursively connected subuniverses, including recursive rules for the compression of a description formulated as rewriting rules, and the expansion of the resulting compressed representation are presented in Wong and Morofsky. ${ }^{1}$

The organization of the PU into pattern classes must satisfy two main criteria. First, each pattern class must be broad enough to encompass the various patterns that are members of the class and display some degree of divergence in their respective descriptions. Second, each pattern class must be adequately selective so that each input pattern can be classified into a unique pattern class. These two criteria can be viewed as the embodiment of a pair of partially conflicting goals, since the first requires an abstraction or generalization of the commonly shared characteristics of all the members of the same pattern class and the second requires a separation of pattern descriptions belonging to different pattern classes based on characteristics that are unique to each class. Of course, the classification of unknown patterns may be based on various degrees of similarity between the input description and the pattern class, depending on (a) the objectives of the classification and (b) the number and similarity of pattern classes in the PU.

When the pattern class "apple" is formed with a sufficiently low threshold on the weighting factors to make both the apple outline and stem essential components, the pattern class is very selective. That is, it makes fewer mistakes in classifying nonapple descriptions as apples.

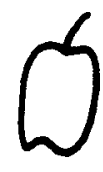

(a)

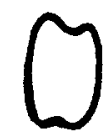

(b)

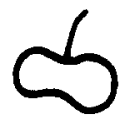

(c)

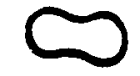

(d)
However, the class is not very general, since it would classify (a) as an apple but not (b), which is missing the stem. Thus, a low threshold tends to increase the selectivity and decreases the generality of a patte $?$ class.

If the class "apple" is formed with a sufficien high threshold. then only the apple outline is made tssential and both (a) and (b) would be classified as apples. This idicates that a high threshold increases generality.
However. selectivity decreases. The pattern of (c) might well be classified as an apple, since its structure matches that of the pattern class and the features of the apple outline, although distorted, also match. It might be considered as a caricature of an apple and would surely be seen as such by many human observers. However, without the stem, as in (d), its resemblance to an apple is much less, even though the two outlines of (c) and (d) are in exact correspondence. But if (c) is classified as an apple, (d) must also be classified as such since the apple outline is the only essential component. Another pattern class might also exist with an essential outline as the only component, such as the pattern class "red blood cell," which also matches (d). Thus, the classification of (d) as an apple or a red blood cell would be uncertain, i.e., selectivity is decreased by the high threshold.

Given an input stereotype, D, the PU can be searched for the set of all pattern classes that have a specified relation to the stereotype. An indefinite number of such sets might be formed by varying the degree of correspondence on different hierarchical levels. For example, one may form the set of all pattern classes of the PU that are identical to D on the first level and that are topologically equivalent on all higher levels. The same method may be used in forming a class from among a group of unknown patterns or in determining the configurational pattern which defines an unknown concept. If Pattern 1 is selected as the stereotype and Patterns 2.6 are presented along with an indication of whether they share in an unknown resemblance to Pattern 1, a commonly shared concept among Patterns 1,2 , and 6 is formed. When these patterns are compared, it is seen that they match in topological equivalence, the least degree of correspondence in PPS. Such techniques can be used to measure the acquisition of simple concepts of form, i.e., those in which the common attributes are dependent on actual stimulus characteristics.

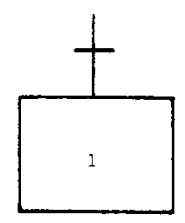

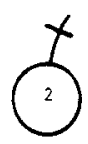

YES

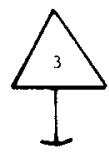

so

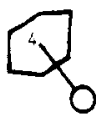

sin

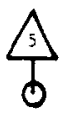

m

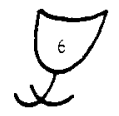

YES

\section{SUMMARY}

PPS describes complex line patterns in terms of the relationships among its components (structural) and the primitive features contained within each of the components. The structural description is size and 
rotation invariant and is used as the initial discriminator in comparisons and classifications, i.e.. if structures do not correspond, there is no need to further compare features. The feature description is dual level in providing an easily accessible partial description and an exhaustive description. This type of description lends itself to efficient processing, especially when multiple representations are more or less possible. Given such descriptions, a variety of comparisons among patterns are possible including the detection of embedded patterns. The buildup of pattern classes is accomplished by recording the occurrence of the various relations until the defining intersection of characteristics emerges. Such techniques are applicable to many familiar psychological studies of the interactions between stimulus structure and learning, e.g., are forms with a less ambiguous structure remembered more easily than those with several ambiguous representations?

\section{REFERENCES}

Djang, $S$. The role of past experience in the visual perception of masked forms. Journal of Experimental Psychology, 1937, 20. 29-59.

Evans, C. R. Fragmentation of patterns occurring with tachistoscopic presentation. Institute of Electrical Engineering Conference Publication, 1968, 42, 250-256.

Falk off, A. D.. \& Iverson, K. E. APL/360: User's manual. Watson Research Center, IBM, 1969.
Freeman, H. Techniques for the digital computer analysis of chain-encoded arbitrary plane curves. Proceedings of the National Electronics Conference, 1961, 17, 421-432.

Heckenmueller, E. G. Stabilization of the retinal image: A review of methods, effects and theory. In R. N. Haber (Ed.), Information processing approaches to visual perception. New York: Holt, Rinehart \& Winston, 1969.

Mackay, D. M. Perception and brain function. In F. O. Schmitt (Ed.), The neurosciences. New York: Rockefeller University Press, 1970.

Merleau-Ponty, M. The phenomenology of perception. London: Routledge \& Kegan Paul, 1962.

Morofsky, E. L. Computer perception of complex line patterns. (Doctoral dissertation, Carnegie-Mellon University) Ann Arbor, Mich: University Microfilms, 1972. No. 72-29,862.

Morofsky, E. L., \& Wong, A. K. C. Computer perception of complex patterns. Second International Joint Conference on Artificial Intelligence, Advance Papers of the Conference, British Computer Society, 1971, 248-257.

Norton, D., \& Stark, L. Eye movements and visual perception Scientific American, June 1971, 224, 35-43.

Pritchard, R. M., Heron, w., Hebb, D. O. Visual perception approached by the method of stabilized images. Canadian Journal of Psychology, 1960, 14, 67-77.

Robinson, J. S. Pamiliar patterns are no easier to see than novel ones. American Journal of Psychology, 1969, 82, 513-522.

1. Wong, A. K. C., \& Morofsky, E, L, Recognition and representation of recursive and quasi-recursive line patterns. npublished manuscript.

2. Morotsky, E. L., \& Wong, A. K. C. Isolating and identifying objects in line drawings. Unpublished manuscript.

3. Wong. A. K. C., \& Morofsky, E. L. Formation and updating of classes of line patterns. Unpublished manuscript. 